\title{
Characterizing electron entanglement in multi-mode mesoscopic conductors
}

\author{
Fabio Taddei*, Vittorio Giovannetti*, Diego Frustaglia ${ }^{\dagger}$ and Rosario Fazio* \\ *NEST CNR-INFM and Scuola Normale Superiore, Piazza dei Cavalieri 7, I-56126 Pisa, Italy \\ $\dagger$ Departamento de Física Aplicada II, Universidad de Sevilla, Av. Reina Mercedes 2, E-41012 \\ Sevilla, Spain
}

\begin{abstract}
We show that current correlations at the exit ports of a beam splitter can be used to detect electronic entanglement for a fairly general input state. First, we demonstrate that multimode entanglement of electrons in a mesoscopic conductor can be detected by a measurement of the zero-frequency current correlations in an electronic Hong-Ou-Mandel interferometer. By this means, one can further establish a lower bound to the entanglement of formation of two-electron input states. Second, we include the situation where electron pairs can enter the beam splitter from the same port or be separated due to backscattering. The proposed scheme allows to discriminate between particle-number and mode entanglement.
\end{abstract}

Keywords: Electronic transport in mesoscopic systems, Entanglement and quantum nonlocality, Entanglement measures, witnesses, and other characterizations

PACS: 73.23.-b, 03.65.Ud, 03.67.Mn

\section{INTRODUCTION}

Generation, manipulation and detection of entangled electrons is necessary for the realization of integrated solid-state quantum computers. Among the several possibilities, a lot of attention has been devoted to the study of entanglement in multiterminal mesoscopic conductors (see Refs. [1,2] for a review). It was shown that entanglement between spatially separated electrons can be detected by means of a beam splitter (BS) [3] which allows the incoming (and possibly entangled) electrons to be interchanged, giving rise to two-particle interference effects. As a result, the symmetry of the incoming state influences the current-noise correlations at the exit ports. In particular, Burkard and Loss [4] derived a lower bound for the entanglement of formation [5] of arbitrary mixed spin states in terms of current-noise correlations. Here we show a generalization of their results to multi-mode input states by introducing the electronic analog of the Hong-Ou-Mandel (e-HOM) [6] optical interferometer. First, we assume that a twoelectron (possibly entangled) input state is injected into a BS whose arms support many propagating modes, including both orbital and spin degrees of freedom. Second, we consider the case where there is a finite probability amplitude that two electrons enter the analyzer at the same input port. This gives rise to two distinct forms of entanglement: occupation-number and mode entanglement $[7,8]$. Under this generalized initial condition the analysis of the entanglement is complicated by super-selection rules induced by particle number conservation $[1,7,8,9]$. We shall show that, for the whole class of twoparticle input states, simple data processing of the measured current cross-correlators can be used to address separately the various entanglement components [10,11].

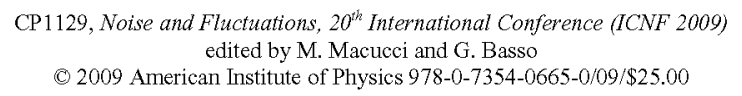




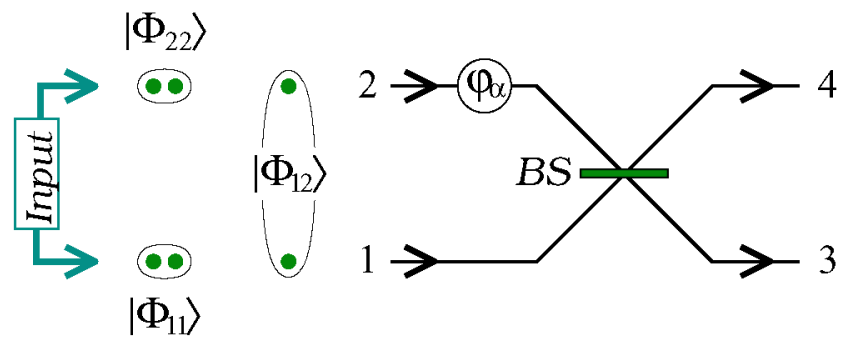

FIGURE 1. Sketch of the electronic Hong-Ou-Mandel interferometer.

\section{THE HONG-OU-MANDEL INTERFEROMETER}

The e-HOM is sketched in Fig. 1. Pairs of electrons of a given energy $E$ above the Fermi sea, prepared in a (possibly entangled) initial state, enter the interferometer from the input ports 1 and 2. Electrons passing through the port 2 undergo an additional, controllable, phase shift $\varphi_{\alpha}$ before impinging on the BS. Zero-frequency current correlations are measured at the output ports 3 and 4 . The electron states, at energy $E$, are labeled by the indices $(j, \alpha)$ where $j=1, \cdots, 4$ labels the ports of the e-HOM interferometer, and where $\alpha$ is the composite index $\{\ell, s\}$ with $s$ referring to electron spin component along the quantization axis, and $\ell$ referring to the orbital channel. Following the LandauerBüttiker scattering formulation of quantum transport [12], the current operator of the $j$-th port is defined as [13]

$$
I_{j}(t) \equiv \frac{e}{h v} \sum_{E, \omega, \alpha} e^{-i \omega t}\left[b_{j, \alpha}^{\dagger}(E) b_{j, \alpha}(E+\hbar \omega)-a_{j, \alpha}^{\dagger}(E) a_{j, \alpha}(E+\hbar \omega)\right],
$$

where $v$ is the density of states of the leads and $b_{j, \alpha}(E)$ are outgoing fermionic operators connected to the incoming $a_{j, \alpha}(E)$ operators via a scattering matrix. The zero-frequency current correlations are defined as

$$
S_{j j^{\prime}}=\lim _{\tau \rightarrow \infty} \frac{h v}{\tau^{2}} \int_{0}^{\tau} d t_{1} \int_{0}^{\tau} d t_{2}\left\langle\delta I_{j}\left(t_{1}\right) \delta I_{j^{\prime}}\left(t_{2}\right)\right\rangle,
$$

where the average $\langle\cdots\rangle$ is taken over the incoming electronic state, $\tau$ is the measurement time and $\delta I_{j}=I_{j}-\left\langle I_{j}\right\rangle$.

\section{ONE ELECTRON PER PORT}

Consider the case in which, for a given energy $E$, one electron per port enters the interferometer from 1 and 2 :

$$
\left|\Phi_{12}\right\rangle=\prod_{E} \sum_{\alpha, \beta} \Phi_{\alpha, \beta} a_{1, \alpha}^{\dagger}(E) a_{2, \beta}^{\dagger}(E)|0\rangle,
$$

where $|0\rangle$ is the Fermi sea at zero temperature and $\Phi_{\alpha, \beta}$ is the two-electron amplitude which we assume to be independent of $E$ and satisfying the normalization 
condition $\sum_{\alpha, \beta}\left|\Phi_{\alpha, \beta}\right|^{2}=1$. A straightforward calculation of the Fano factor $F_{j j^{\prime}}=$ $S_{j j^{\prime}} /\left(2 e \sqrt{\left\langle I_{j}\right\rangle\left\langle I_{j^{\prime}}\right\rangle}\right)$ leads to the expression

$$
F_{34}=-T(1-T)\left(1-w_{\Phi}\right)
$$

where $T$ is the transmissivity of the BS and

$$
w_{\Phi} \equiv \sum_{\alpha, \beta}\left[\Phi_{\alpha, \beta}\right]^{*} \Phi_{\beta, \alpha} e^{i\left(\varphi_{\alpha}-\varphi_{\beta}\right)}
$$

is a (real) quantity which depends on the controllable set of phases $\left\{\varphi_{\alpha}\right\}$. By using the Chauchy-Schwartz inequality and the normalization condition of $\Phi_{\alpha, \beta}$ it follows that for a generic input state (3) one has $-1 \leq w_{\Phi} \leq 1$. However, if $\left|\Phi_{12}\right\rangle$ in Eq. (3) is a separable state with respect to the input ports 1 and 2 , it is possible to show that $w_{\Phi}$ is non negative. Negative values of $w_{\Phi}$ are hence a direct evidence of the presence of entanglement in the input state. The quantity $w_{\Phi}$ therefore acts as an entanglement witness [14] for the class of two-electron states analyzed here. Furthermore, it can be proven $[4,10]$ that the Fano factor of Eq. (4) is related to the entanglement of formation $E_{f}$ of the input state $\rho$ of the e-HOM interferometer by the inequality

$$
E_{f}(\rho) \geq \mathscr{E}(\bar{W}(\rho))
$$

with

$$
\bar{W}(\rho)=-\frac{F_{34}}{2 T(1-T)},
$$

and $\mathscr{E}(x)=H\left(\frac{1}{2}+\sqrt{x(1-x)}\right)$ for $x \in[1 / 2,1]$ and null otherwise [here $H(x)=$ $\left.-x \log _{2} x-(1-x) \log _{2}(1-x)\right]$. This shows that by measuring the Fano factor one can determine a lower bound for the entanglement of formation through Eq. (6).

\section{GENERIC INPUT STATE}

Let us now consider a generic input state of the form

$$
|\Psi\rangle=\sin \theta\left(\cos \phi\left|\Phi_{11}\right\rangle+\sin \phi\left|\Phi_{22}\right\rangle\right)+\cos \theta\left|\Phi_{12}\right\rangle,
$$

where $\theta, \varphi \in[0, \pi / 2]$ and $\left|\Phi_{i j}\right\rangle$ describe two electrons of energy $E$ entering the BS from the $i$-th and $j$-th port respectively $(i, j=1,2)$. The analysis of the entanglement contained in the state $|\Psi\rangle$, can be done naturally by bipartitioning the system with respect to the port labels 1 and 2 . The case $\theta=0$ has been considered in the previous section. For $\theta \neq 0$ we have both occupation-number and mode entanglement. The first one is present whenever we have a non trivial superposition among terms where each lead supports a different number of incoming electrons, i.e. $\left|\Phi_{11}\right\rangle,\left|\Phi_{22}\right\rangle$, and $\left|\Phi_{12}\right\rangle$. The second one, instead, originates from the component $\left|\Phi_{12}\right\rangle$ of Eq. (8): it is present when the leads 1 and 2 possess one electron each and are entangled through the electronic orbital/spin modes $\alpha$. The (dimensionless) current cross-correlator at the output ports 3 and 4 is

$$
C_{34} \equiv \frac{h^{2} v^{2}}{2 e^{2}} \lim _{\tau \rightarrow \infty} \int_{0}^{\tau} d t_{1} d t_{2} \frac{\left\langle I_{3}\left(t_{1}\right) I_{4}\left(t_{2}\right)\right\rangle}{\tau^{2}} .
$$


Differently from $S_{34}$, the $C_{34}$ is linear in the input state of the system for $\theta \neq 0$. For any given density matrix $\rho=\sum_{\ell} p_{\ell}\left|\Psi_{\ell}\right\rangle\left\langle\Psi_{\ell}\right|$ with $\left|\Psi_{\ell}\right\rangle$ as in Eq. (8) we obtain $C_{34}(\rho)=\sum_{\ell} p_{\ell} C_{34}\left(\Psi_{\ell}\right)$, where the correlators $C_{34}\left(\Psi_{\ell}\right)$ take the form

$$
C_{34}(\Psi)=\left[1+w \cos ^{2} \theta+v \sin ^{2} \theta \sin (2 \phi)\right] / 4 .
$$

Here, $w$ and $v$ are real quantities satisfying $|w|,|v| \leq 1$ which depend upon the interferometer phases $\varphi_{\alpha}$ and the input state parameters. Following the derivation of Refs. $[4,10]$, it is easy to show [11] that $E_{f}(\rho)$ can be lower bounded as in Eq. (6) by the quantity

$$
\bar{W}(\rho)=1-2 C_{34}(\rho) .
$$

It follows that $C_{34}(\rho)<1 / 4$ implies $E_{f}(\rho)>0$. Therefore, we can conclude that also in the general case in which the two electrons can enter the same port, values of $C_{34}$ smaller than $1 / 4$ are direct evidence of entanglement in the input state. It is also possible to discriminate between occupation-number and mode entanglement through the quantities $C_{34}^{(+)}(\Psi)=\left[1+w \cos ^{2} \theta\right] / 4$ and $C_{34}^{(-)}(\Psi)=v \sin ^{2} \theta \sin (2 \phi) / 4$. The presence of mode entanglement can be detected by finding values of $\varphi_{\alpha}$ such that $C_{34}^{(+)}<1 / 4$. Vice-versa, we observe that any value of $C_{34}^{(-)}$different from zero is indicative of occupation-number entanglement in the system.

\section{REFERENCES}

1. C. W. J. Beenakker, "Electron-hole entanglement in the Fermi sea", in International School of Physics Enrico Fermi, Vol. 162, Quantum Computers, Algorithms and Chaos, edited by G. Casati et al., IOS Press, Amsterdam, 2006, pp. 307???347.

2. G. Burkard, "Theory of solid state quantum information processing", in Handbook of Theoretical and Computational Nanotechnology, edited by M. Rieth and W. Schommers, American Scientific Publisher, Valencia, 2006.

3. G. Burkard, D. Loss, and E.V. Sukhorukov, Phys. Rev. B 61, R16303-R16306 (2000).

4. G. Burkard, and D. Loss, Phys. Rev. Lett. 91,087903 (2003).

5. C. H. Bennett, D. P. DiVincenzo, J. A. Smolin, and W. K. Wootters, Phys. Rev. A 54, 3824-3851 (1996); W. K. Wootters, Quantum Inf. Comput. 1, 27-44 (2001).

6. C. K. Hong, Z. Y. Ou, and L. Mandel, Phys. Rev. Lett. 59, 2044-2046 (1987).

7. H. M. Wiseman and J. A. Vaccaro, Phys. Rev. Lett. 91,097902 (2003); H. M. Wiseman, S. D. Bartlett, and J. A. Vaccaro, quant-ph/0309046.

8. N Schuch, F. Verstraete and J. I. Cirac, Phys. Rev. Lett. 92, 087904 (2004); Phys. Rev. A 70, 042310 (2004).

9. F. Verstraete and J. I. Cirac, Phys. Rev. Lett. 91, 010404 (2003).

10. V. Giovannetti, D. Frustaglia, F. Taddei, and R. Fazio, Phys. Rev. B 74, 115315 (2006).

11. V. Giovannetti, D. Frustaglia, F. Taddei, and R. Fazio, Phys. Rev. B 75, 241305 (2007).

12. M. Büttiker, Phys. Rev. B 46, 12485-12507 (1992).

13. G B. Lesovik, Pis'ma Zh. Eksp. Teor. Fiz. 49, 513-515 (1989) [ JETP Lett. 49, 592-594 (1989)].

14. M. Horodecki, P. Horodecki, and R. Horodecki, Phys. Lett. A 223, 1-8 (1996); M. Lewenstein, B. Kraus, J. I. Cirac, and P. Horodecki, Phys. Rev. A 62, 052310 (2000). 